\section{Implementación de la plataforma Open Journal Systems (OJS) en el Journal Boliviano de Ciencias (JBC)}

Resumen: El objetivo del presente estudio es analizar los primeros resultados de la implementación del Open Journal Systems (OJS) en la revista Journal Boliviano de Ciencias (JBC). Se utilizaron datos históricos de la publicación del JBC de los años 2019, 2020 y 2021, incluyendo aquellos proporcionados por el sistema OJS. Los resultados muestran que ha aumentado la visibilidad de la revista y la recepción de artículos científicos por parte de instituciones externas, alcanzando el $43,75 \%$ del total de publicaciones. El proceso editorial es fluido y permite ser registrado para dar seguimiento. A pesar de todas las mejoras que trae la implementación del OJS, todavía debe consolidarse para posteriormente atender otros desafíos del JBC.

Palabras clave: Visibilidad. Artículo científico. Proceso editorial.

Open Journal Systems (OJS) platform Implementation in the Journal Boliviano de Ciencias (JBC)

Abstract: This study aims to analyze the first results of the implementation of the Open Journal Systems (OJS) in the Journal Boliviano de Ciencias (JBC). Historical data from the JBC publication for the years 2019, 2020 and 2021 were used, including those provided by the OJS system. The results show that the visibility of the journal and the reception of scientific articles by external institutions has increased, reaching $43.75 \%$ of all publications. The editorial process is fluid and allows to be registered for follow-up. Despite all the improvements that the OJS implementation brings, it still needs to be consolidated to later address other JBC challenges.

Keywords: Visibility. Scientific article. Editorial process.

\section{Implementação da plataforma Open Journal Systems (OJS) no Journal Boliviano de Ciencias (JBC)}

Resumo: O objetivo deste estudo é analisar os primeiros resultados da implantação do Open Journal Systems (OJS) no Journal Boliviano de Ciencias (JBC). Foram utilizados os dados históricos da publicação do JBC para os anos de 2019, 2020 e 2021, inclusive os fornecidos pelo sistema OJS. Os resultados mostram que a visibilidade da revista e a recepção de artigos científicos por instituições externas tem aumentado, atingindo $43,75 \%$ do total de publicações. O processo editorial é fluido e permite ser cadastrado para acompanhamento. Apesar de todas as melhorias que a implantação do OJS traz, o sistema ainda precisa ser consolidado para posteriormente enfrentar outros desafios do JBC.

Palavras-chave: Visibilidade. Artigo científico. Processo editorial.

\section{JOAQUIN HUMBERTO} AQUINO ROCHA

Rio de Janeiro, Rio de Janeiro, Brasil

Universidade Federal de

Rio de Janeiro

Universidad Privada del Valle

\section{EDSON GASTON} MONTAÑO BAUTISTA

Rio de Janeiro, Rio de Janeiro, Brasil

Universidade Federal de Rio de Janeiro

Universidad Privada del Valle

\section{DAYSI LIDIA IÑIGUEZ CALVETI}

Tiquipaya, Cochabamba, Bolivia

Universidad Privada del

Valle 


\section{Introducción}

La revista científica es el canal de comunicación más utilizado tanto por académicos como investigadores, siendo el artículo científico el recurso informativo más importante. Inicialmente, las revistas científicas solo estaban disponibles en edición impresa y varias, incluso hasta ahora, requieren costosas subscripciones, generando inconvenientes de acceso a la información. Esto ha llevado, en los últimos años, a la creación de revistas electrónicas que facilitan el proceso editorial y se relacionan con el acceso abierto (SANDES-GUIMARÃES; COSTA, 2012).

Las revistas electrónicas utilizan diversos sistemas para publicar su contenido, uno de los más extendidos internacionalmente es el Open Journal Systems (OJS). El OJS es un sistema de gestión y publicación de revistas científicas en línea, permite realizar un seguimiento detallado de los manuscritos, disminuye el tiempo en el proceso editorial y facilita la interacción entre todos los participantes. En OJS el manejo de artículos es eficiente y unificado, cubriendo todas las etapas, desde la recepción hasta la publicación (PINA; SÁNCHEZ, 2010). El sistema es de código abierto y fue desarrollado por el grupo Public Knowlegde Proyect (PKP) en Canadá, con el fin de desarrollar tecnología para la investigación científica en el área de gestión, impulsando el acceso abierto (PKP, 2021).

El Journal Boliviano de Ciencias (JBC) es una revista científica que inició en formato impreso, para luego tener su versión digital, pero esto solo se limitaba a proporcionar el Portable Document Format (PDF) en línea. Desde el año 2021, se empezó a utilizar el OJS a fin de dar mayor visibilidad y añadir mejoras a la revista, como se recomienda en la literatura (NDUNGU, 2020).

Previa implementación del OJS en el JBC, el comité editorial optó por este sistema para solucionar algunos problemas de la revista, como ser: visibilidad, solo difundida a nivel local y nacional; sistematización de la gestión editorial, realizada únicamente por correo electrónico entre autores, revisores y editores; optimización de los procesos de edición y publicación, que no estaban conectados con las otras etapas, y aumentar la recepción de artículos que no sean de la propia institución.

En este sentido, el propósito del presente trabajo es dar a conocer los primeros resultados de la implementación del OJS en el JBC, como parte de las mejoras propuestas en estudios previos (ROCHA; BAUTISTA-MONTAÑO; IÑIGUEZ-CALVETI, 2020). Considerando que las revistas científicas se encuentran en un proceso de perfeccionamiento continuo (HAUSMANN; MURPHY, 2016). Se reportan las ventajas de la 
implementación del OJS en la gestión editorial: metadatos, licencias, integración a bases de datos, etc.; además de las dificultades presentadas durante el proceso.

\section{Metodología}

El presente estudio es de naturaleza descriptiva e histórica, buscando realizar un análisis de la implementación del OJS versión 3.2.1.4 en el JBC, revista científica de la Facultad de Tecnología de la Universidad Privada del Valle, relatando la experiencia a fin de crear un registro y proponer mejoras en el futuro.

Se utilizaron datos históricos de publicación de los últimos números del JBC (2019, 2020 y 2021): cantidad de publicaciones, artículos recibidos y origen de los estudios además de la información proporcionada por el OJS, como el número de consultas al resumen y visualizaciones.

\section{Resultados y discusión}

La primera fase consistió en la instalación y personalización del sitio web de la revista, siendo alojada en el portal de revistas Univalle (https://revistas.univalle.edu/). Desde abril de 2021 se procedió a publicar por medio de OJS, iniciando con un número especial y, en junio, se publicó el primer número oficial (№50).

Si bien el JBC es publicado en forma impresa y digital, al no poseer un sistema de gestión editorial y publicación, no se podía conocer el impacto y/o visibilidad de los artículos. No obstante, el OJS permite ver el detalle de las consultas al resumen y visualizaciones de los archivos publicados. La Figura 1 muestra la evolución del acceso a los artículos publicados desde el primer número publicado en OJS.

Esto se debe a que el OJS permite que la revista se indexe automáticamente en Google Scholar, permitiendo su fácil detección (NDUNGU, 2020).

La Figura 2 muestra el detalle de los artículos publicados en los años 2019, 2020 y 2021, comparando la distribución de artículos publicados por institución (propia, otra nacional e internacional).

Se puede observar que la cantidad de artículos publicados aumentó en el 2021, año de implementación del OJS, todavía faltando un número. Esto claramente está relacionado con el número de envíos, 31 hasta la fecha, duplicando a los años anteriores (2019 y 2020).

Figura 1 - Consultas al resumen y visualizaciones de los artículos publicados 


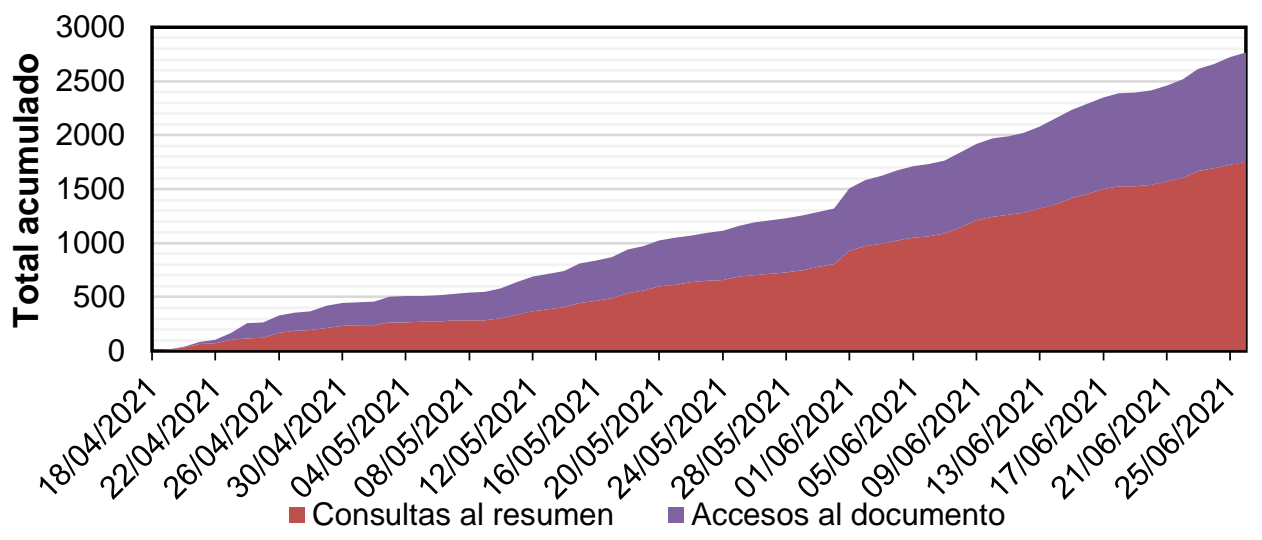

Fuente: Elaboración de los autores (2021).

Figura 2 - Detalle de las publicaciones (2019, 2020 y 2021)

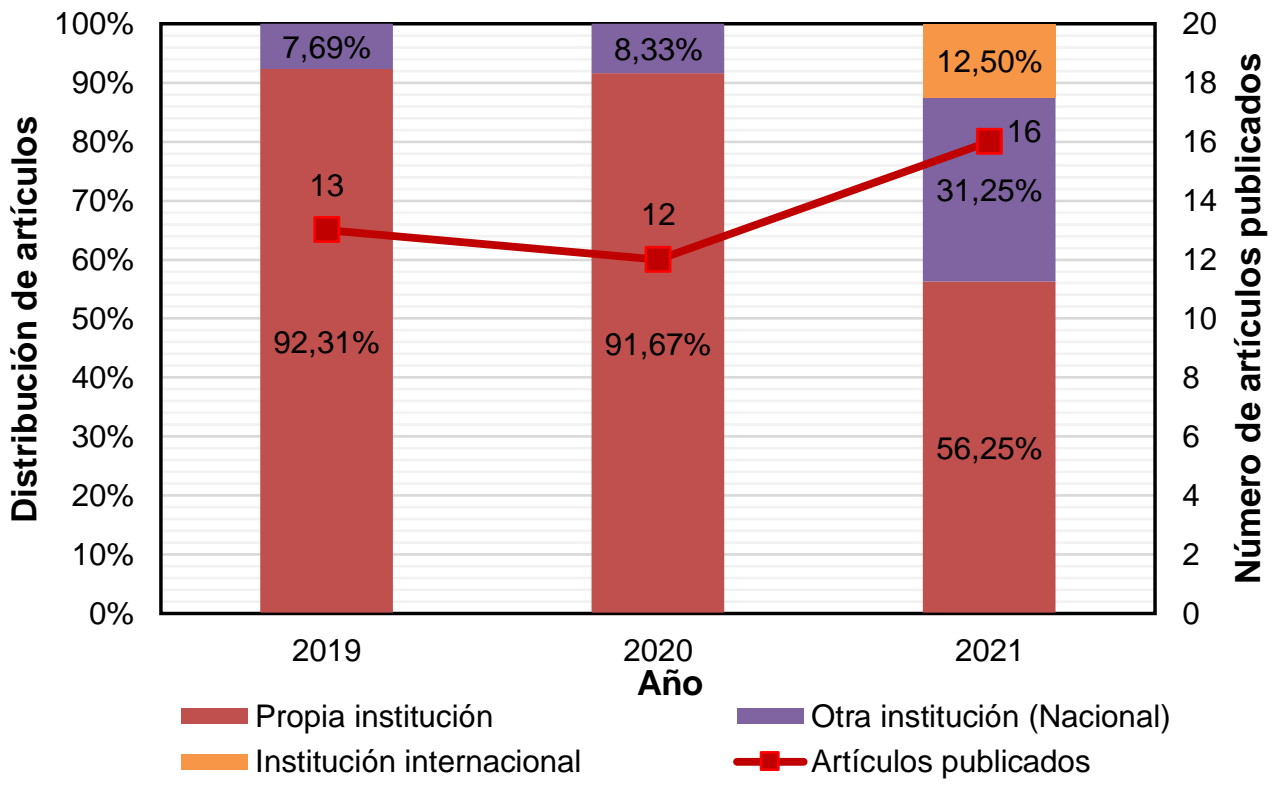

Fuente: Elaboración de los autores (2021).

Un desafío que tiene el comité editorial del JBC es ampliar la cantidad de publicaciones de instituciones externas (nacionales e internacionales). En la Figura 2 se observa que, este porcentaje aumentó a $43,75 \%$ comparado con el 7,69\% y $8,33 \%$ en los años 2019 y 2020, respectivamente. Se tiene la publicación de trabajos provenientes de instituciones externas, cinco nacionales $(31,25 \%)$ y dos internacionales $(12,50 \%)$, demostrando que la visibilidad de la revista ha aumentado sustancialmente con el OJS.

Respecto a la gestión editorial, el OJS permite que los contenidos se gestionen íntegramente en línea y que exista asignación de roles, lo que ayuda a conectar todo el proceso editorial (gestor de la revista, editor, editor de sección, revisor, autor, corrector, diagramador, entre otros). Adicionalmente se garantiza el envío directo de los manuscritos y se logra una interfaz multilingüe (CASCARET SOTO et al., 2017). Pero también mediante

Esta obra está sob licença Creative Commons Atribuição 4.0 Internacional 
la implementación del OJS se lograron realizar acciones y añadir elementos con los que no contaba la revista: gestión de metadatos; políticas de derechos de autor y licencias; integración de la revista con Open Researcher and Contributor ID (ORCID); asignación de Digital Object Identifier (DOI); exportación de datos a Crossref, entre otros.

Sin embargo, se reportaron dificultades durante el proceso de implementación. Como toda herramienta informática se requiere una inversión de tiempo para aprender las bondades y el mecanismo de funcionamiento del sistema. Para algunos editores de sección esto representó un proceso complejo, sobre todo por estar acostumbrados al modelo anterior, pero al final lograron manejar adecuadamente el sistema. Los autores y revisores también mostraron algunos problemas al no estar familiarizados con el sistema, lo que derivó en envíos con errores y/o incompletos, problemas que fueron subsanados en el proceso de publicación.

Para lograr una mayor aceptación del sistema por todos los participantes, se proponen algunas medidas: organizar cursos de capacitación destinados a autores, revisores, futuros editores, entre otros; preparar manuales de uso del sistema, disponible en el sitio de la revista, y habilitar un correo electrónico de soporte, dedicado a resolver dudas sobre el manejo de sistema.

Finalmente, es importante mencionar que, en Bolivia, existen desafíos respecto a la publicación de revistas científicas, no se tienen lineamientos generales y la mayoría publica en PDF como versión digital, como era el caso del JBC, muy pocas utilizan OJS. Adicionalmente no existe una estructura nacional que permita seleccionar o recomendar revistas científicas para publicar trabajos de investigación. Sin embargo, es necesario realizar cambios que permitan utilizar sistemas que no solo beneficien el proceso editorial, sino mejoren la visibilidad de las publicaciones y estén disponibles en acceso abierto.

\section{Consideraciones finales}

Las diferentes herramientas electrónicas, en este caso el OJS, proporcionan facilidades para desarrollar un eficiente proceso editorial y dar mayor visibilidad a los trabajos publicados. En el poco tiempo de implementación del OJS en el JBC, se ha logrado cumplir con las expectativas del comité editorial, que incluye herramientas necesarias para publicar artículos en acceso abierto y permitiendo la internacionalización de la revista.

Si bien el OJS ha mejorado varios aspectos del JBC, todavía estos cambios están consolidándose y quedan otros desafíos, como lograr mayores indexaciones. El proceso de 
implementación también ha permitido identificar las necesidades respecto a la gestión editorial, para lo cual se proponen acciones de mejora.

Esta experiencia puede servir como ejemplo para otras revistas bolivianas, las cuales pueden considerar los aspectos mencionados para publicar resultados de investigación de forma gratuita, disponible y eficiente, mediante una mejor gestión editorial.

\section{Referencias}

CASCARET SOTO, X.; PARDO GOMEZ, M. E.; IZQUIERDO LAO, J. M.; ACOSTA ORTEGA, L. Medisan, v. 21, n. 11, p. 3285-3290, 2017.

HAUSMANN, L.; MURPHY, S. P. The challenges for scientific publishing, 60 years on. Journal of Neurochemistry, v. 139, n. 52, p. 280-287, 2016. DOI:

https://doi.org/10.1111/jnc. 13550

NDUNGU, M. W. Publishing with Open Journal Systems (OJS): A Librarian's Perspective. Serials Review, v. 46, n.1, p. 21-25, 2020. DOI:

http://doi.org/10.1080/00987913.2020.1732717

PINA, F. H.; SÁNCHEZ, J. J. M. Indicadores de calidad de las revistas científicas y sistema de gestión editorial mediante OJS. Revista de Investigación Educativa, v. 28, n.1, p. 13-29, 2010.

PUBLIC KNOWLEGDE PROYECT - PKP. Open Journal Systems, 2021. Disponible en: https://pkp.sfu.ca/ojs/

ROCHA, J. H. A.; MONTAÑO-BAUTISTA, E. G.; IÑIGUEZ-CALVETI, D. L. Acciones de mejora en el proceso editorial científico del Journal Boliviano de Ciencias: estudio de caso. In: ABEC Meeting Live, 2020. Anais... São Paulo: Associação Brasileira de Editores Científicos, 2020. DOI: http://dx.doi.org/10.21452/abecmeeting2020.01

SANDES-GUIMARÃES, L. V. D.; COSTA, S. M. D. S. Brazilian scientific journals that use the Open Journal Systems (OJS): a quality analysis. JISTEM-Journal of Information Systems and Technology Management, v. 9, n. 1, p. 61-88. 2012. DOI: http://doi.org/10.4301/S1807-17752012000100004 
Joaquin Humberto Aquino Rocha

Rio de Janeiro, Rio de Janeiro, Brasil

Universidade Federal de Rio de Janeiro

Mini currículum: Graduado en Ingeniería Civil por la Universidad Mayor de San Simón - UMSS (Bolivia). Maestría en Ingeniería Civil por la Universidade de Pernambuco - UPE (Brasil). Estudiante de doctorado en Ingeniería Civil en la Universidade Federal de Rio de Janeiro y Editor de sección en Ingeniería Civil del Journal Boliviano de Ciencias.

Contribución de autoría: Conceptualización, Curación de datos, Análisis formal, Supervisión, Validación, Visualización, Redacción (borrador original) y Redacción (revisión y edición).

ORCID ID: https://orcid.org/0000-0002-3383-6379

Lattes: http://lattes.cnpq.br/1787659871780888

E-mail: joaquinaquinorocha@gmail.com

\section{Edson Gaston Montaño Bautista}

Rio de Janeiro, Rio de Janeiro, Brasil

Universidade Federal de Rio de Janeiro

Mini currículum: Graduado en Ingeniería Mecánica y de Automatización Industrial por la Universidad Privada del Valle (Bolivia). Estudiante de maestría en Ingeniería Eléctrica en la Universidade Federal de Rio de Janeiro y Editor de sección de Electromecánica del Journal Boliviano de Ciencias.

Contribución de autoría: Investigación, Administración del proyecto, Recursos, Visualización, Redacción (borrador original) y Redacción (revisión y edición).

ORCID ID: https://orcid.org/0000-0002-7105-9072

Lattes: http://lattes.cnpq.br/6877310844638939

E-mail: mbautistaedson@gmail.com

\section{Daysi Lidia Iñiguez Calveti}

Tiquipaya, Cochabamba, Bolivia

Universidad Privada del Valle

Mini currículum: Graduada en Ingeniería Industrial por la Universidad Mayor de San Simón - UMSS (Bolivia). Maestría en Dirección de Proyectos de la Universidad Rey Juan Carlos (España). Coordinadora del Departamento de Industrias de la Universidad Privada del Valle y Editora de sección en Industrias y Alimentos del Journal Boliviano de Ciencias.

Contribución de autoría: Investigación, Administración del proyecto, Recursos, Visualización, Redacción (borrador original) y Redacción (revisión y edición).

ORCID ID: https://orcid.org/0000-0001-8101-0427

Lattes: http://lattes.cnpq.br/8627624128330466

E-mail: diniguezc@univalle.edu 